\title{
Estereotipos negativos sobre vejez y el bienestar psicológico del adulto mayor.
}

\section{Negative stereotypes about old age and the psychological well-being of the elderly.}

\section{Estereotipos negativos - bienestar psicológico}

\author{
Hugo Sánchez Rogel. Ps.Cl. ${ }^{(1)}$ \\ María Lazo Moreira Ps.Cl. ${ }^{(2)}$
}

${ }^{1}$ Universidad Técnica de Manabí, Instituto de Posgrado, Maestría Académica con Trayectoria de Investigación en Psicología mención Psicoterapia, Manabí-Ecuador. email: lsanchez1578@utm.edu.ec

${ }^{2}$ Universidad Técnica de Manabí, Facultad de Ciencias Humanísticas y Sociales, Manabí-Ecuador. email: vlazo.moreira@utm.edu.ec

Contacto: 1sanchez1578@utm.edu.ec

\section{Resumen}

La presente investigación gira entorno a la afectación que tiene la presencia de estereotipos negativos en la familia sobre el bienestar psicológico de las personas adultas mayores. El objetivo fue identificar la incidencia de los estereotipos negativos sobre la vejez en la familia en el bienestar psicológico de los adultos mayores. Se aplicó el cuestionario de Estereotipos Negativos de la Vejez (CENVE) a 20 familias correspondientes a 20 adultos mayores, a los cuales se les aplicó la Escala de Bienestar Psicológico (Carol Ryff- 1995). Los resultados indican que existe una asociación estadística entre los estereotipos negativos sobre la vejez en la familia y el bienestar psicológico de los adultos mayores, validado con prueba del Chi cuadrado $\left(x^{2}(6)=24,248, p<0,05\right)$. Se concluye que existe relación proporcional de incidencia de los estereotipos negativos hacia la vejez por parte de la familia con el bienestar psicológico del adulto mayor.

Palabras clave: Estereotipos, prejuicios, viejismo, bienestar psicológico, gerontología

\section{Abstract}

The present investigation revolves around the affectation that the presence of prejudices and stereotypes in the family environment has on the psychological well-being of older adults. The objective was to identify the incidence of negative stereotypes about old age in the family environment on the psychological well-being of older adults. The Negative Stereotypes of Old Age questionnaire (CENVE-2005) was applied to 20 families 
corresponding to 20 older adults, to whom the Psychological Well-being Scale was applied (Carol Ryff-1995). The results indicate that there is a statistical and directly proportional association between negative stereotypes towards old age by the family and the psychological well-being of the elderly.

Key words: Stereotypes, prejudices, ageism, psychological well-being, gerontology

\section{Introducción}

Los estereotipos son creencias generalizadas y subjetivas sobre lo que se supone son rasgos inherentes de un determinado grupo social (Franco S et al., 2010a). La investigación en el campo de la vejez destaca la caracterización negativa asociada al deterioro de salud, dependencia, inseguridad económica, soledad y decadencia en las capacidades físicas y verbales (Rodríguez, 2019). Pero también podrían presentarse estereotipos positivos, resaltando la sabiduría y experiencia, o neutrales de acuerdo al contexto particular (Velasco et al., 2018).

Los estudios que recogen la imagen social de las personas adultas mayores empiezan a surgir a mediados del siglo pasado, en una serie de trabajos realizados por profesionales de la medicina y la psiquiatría con personas que fueron seleccionadas generalmente a partir de consultas médicas, de los hospitales y de los centros psiquiátricos, por lo tanto, estos no representaban a la generalidad de la población de personas adultas mayores, sino que eran, más bien, exponentes de la vejez patológica. No obstante, de los resultados de estos trabajos, se infirieron conclusiones para el conjunto de la población mayor, lo que ha favorecido un patrón generalizado de creencias consideradas normativas en la vejez, tales como: "los adultos mayores son obstinados", "ya no pueden aprender", "son inútiles", "es normal estar deprimido o enfermo si estás viejo", "las relaciones sexuales ya no son para los ancianos", "los adultos mayores son como niños”, entre otras (Sánchez, 2004, p.21).

Blair y Banaji (1996, citado en Sánchez, 2004) sugieren que la activación cognitiva del estereotipo es un proceso automático e involuntario, mientras que la aplicación del mismo se supone un proceso que está bajo el control de los sujetos, es decir que un esfuerzo intencional o controlado por parte del observador puede soslayar las expresiones conductuales anexas al pensamiento estereotipado (p.44).

Aunque en las últimas décadas se ha realizado una variedad de estudios relacionados al impacto de los estereotipos sociales en diferentes grupos humanos, por razón de la orientación sexual, religión, raza o el género, entre los más nombrados, los trabajos que se han hecho con muestras de adultos mayores son mucho menos numerosos (Sánchez, 2004). Al contrario de los prejuicios racistas o sexistas, en los etarios no se evidencia un odio ni vergüenza en las personas que los sostienen como se pone de manifiesto en el hecho de que la sociedad no sanciona de igual forma a los que expresan estos estereotipos, a quienes sustentan estigmas hacia la vejez (Levy y Banaji, 2002, citado en Sánchez, 2004, p.78).

Una diferencia de los estereotipos hacia la vejez en relación a los de otro tipo, es que todas las personas están destinadas a envejecer y son propensas a padecer las consecuencias de los propios prejuicios y del círculo 
social, lo que hace primordial generar nuevos aportes investigativos en la temática (Palmore,1982, citado en Sánchez, 2004, p.78).

Otra consecuencia de los estereotipos negativos hacia la población adulta mayor, es la exclusión de la sociedad, de la comunidad y la vida familiar, muchas veces las prácticas prejuiciosas son invisibilizadas, que no son percibidas como tales y otras veces naturalizadas, que se aceptan como "normales" y esperables tanto por parte de quien discrimina como de quien es discriminado (Instituto Nacional contra la Discriminación, la Xenofobia y el Racismo, s. f.). Estas creencias tienen un valor social y legal que trastoca de manera significativa en el bienestar psicológico de las personas (Córdoba et al., 2010).

El bienestar psicológico es un estado de equilibrio entre aspectos físicos, psicológicos y sociales, así como el dominio que el sujeto tiene sobre su entorno, también implica la presencia de procesos positivos, en relación a la satisfacción y la calidad de vida de acuerdo a los propios criterios del individuo (Ryff, 1995, citado en García \& Ibarra, 2018, p.6).

De igual manera, en el bienestar psicológico intervienen características personológicas que, sumado al aspecto cognitivo y circunstancias medio ambientales, generan una carga valorativa en los logros alcanzados y una evaluación de expectativas sobre una serie de dominios vitales sobre la satisfacción con su vida en general (Guerra, 2014); la percepción del bienestar psicológico se verá influenciada por las constructos sociales y personales, además de las diferentes estrategias de afrontamiento que hayan generado en su historia de vida, pero siempre tomando en cuenta que el adulto mayor se desenvuelve dentro de una sociedad y en constante interacción con otros miembros de su entorno (Ortiz Arriagada \& Castro Salas, 2009).

La familia como red social de apoyo, acrecienta su importancia con el envejecimiento de sus integrantes de más edad que ameriten asistencia dentro de las actividades de la vida diaria, las tareas de cuidado y apoyo que requieren las personas adultas mayores con dependencia funcional generan alteraciones en la dinámica al interior de las familias, la cual se ve obligada a modificarse al momento que se debe asumir roles asistenciales por parte de los miembros de la familia, cambiando la estructura y composición de la misma (Montes \& Hebrero, 2008).

Las relaciones familiares con el adulto mayor están determinadas por múltiples factores: unos de tipo material; como incentivos económicos, garantía de servicios básicos, eliminación de barreras arquitectónicas, etc; y otros psicológicos como las necesidades de afecto, cuidados de salud, apoyo, seguridad, desarrollo de expectativas hacia el futuro, entre otros (Valhondo et al., s. f.)., generando así un espacio familiar que permita el bienestar psicológico (Estrada et al., 2008). El desconocimiento y deformación de las potencialidades de las personas adultas mayores, constituye el primer paso para la discriminación y la incorporación de los prejuicios tanto a nivel familiar como visión personal del adulto mayor (Pabón \& Delgado, 2017). 
Córdoba et al. (2010) expresan que es muy frecuente que los adultos mayores proyecten sobre sí mismos tales estereotipos ya que estos han sido generados durante el transcurso de su vida, lo cual se refleja en su comportamiento, de igual manera las familias cuidadoras ven a las personas mayores como su propio envejecimiento, reforzando estas ideas erróneas en los miembros de la familia, un ciclo que perdura en el imaginario por generaciones, este principio se ha denominado "la profecía que se autocumple" (p.76).

Cuando la persona adulta mayor alcanza un grado de dependencia, se enfrenta a condiciones que propician la vulnerabilidad disminuyendo la autoeficacia (Pabón \& Delgado, 2017), y cerca del 80\%-88\% de los cuidados que recibe un adulto mayor, de los cuales dependerá proporcionalmente su bienestar psicológico, son proporcionados exclusivamente por la familia, la cual suele ser multigeneracional (Aparicio et al., 2008).

A pesar de que se han realizado estudios comparativos sobre la presencia de estereotipos negativos por grupos etarios, han sido en población sin relación filial, es decir no se han considerado a todos los miembros convivientes del adulto mayor y su bienestar psicológico, por lo anterior expuesto, se plantea como principal objetivo determinar la incidencia de los estereotipos negativos de la familia sobre la vejez en el bienestar psicológico de las personas adultas mayores, con el propósito de generar datos que contribuyan al estudio integral de la vejez e incrementar conocimientos referentes a las creencias que envuelven la interacción familiar con el adulto mayor. De manera más concreta con el presente trabajo se pretende dar respuesta a los siguientes objetivos específicos: (1) Describir el nivel de presencia de estereotipos negativos que tiene la familia sobre la vejez; (2) Determinar la preponderancia factorial de acuerdo al CENVE; (3) Identificar cómo afectan los estereotipos negativos de la familia al bienestar psicológico de los adultos mayores.

\section{Materiales y métodos}

La investigación es de corte descriptivo transversal, fue dirigida a 20 adultos mayores y sus respectivas familias, pertenecientes al programa Mis mejores años del Ministerio de Inclusión Económica y Social, en el cantón 24 de Mayo, Manabí, Ecuador. La muestra fue de grupos homogéneos, bajo los siguientes criterios de inclusión: que la persona tenga 65 años o más y se encuentre en condición socioeconómica baja. Es preciso mencionar que los adultos mayores poseen discapacidad (física y/o auditiva). Se excluyen los adultos mayores que presenten deterioro cognitivo grave, que vivan solos o se nieguen a participar.

Previo consentimiento informado, se aplicó a los familiares del adulto mayor, el Cuestionario de Estereotipos Negativos hacia la Vejez (CENVE) de Blanca y Torres (2005), con 15 items tipo Likert que explora creencias del sujeto, tiene tres dimensiones: Salud, Motivación Social y Carácter-personalidad.

Para evaluar el bienestar psicológico de los adultos mayores, se les aplicó la Escala de bienestar psicológico de Ryff (1995) validado para Colombia, que se encuentra constituido por 6 dimensiones las cuales son: Autoaceptación, Dominio del entorno, Relaciones positivas, Crecimiento personal, Autonomía y Propósito en la vida. 
Los datos se analizaron en SPSS V25.0 para determinar la correlación estadística entre las variables usando la prueba Chi Cuadrado de Pearson y frecuencias globales y por factores, no existieron conflictos de interés y los datos personales de los participantes se manejaron bajo criterio de confidencialidad.

\section{Resultados}

Con respecto al primer objetivo de la investigación se evaluó a los familiares de los adultos mayores con el Cuestionario CENVE, el análisis de la escala global dio como resultado 55,0\% de familiares con nivel alto en estereotipos negativos hacia la vejez, siendo la categoría más elevada seguida del nivel moderado y el nivel bajo (tabla 1), así mismo se adjuntan los puntajes por categoría de la escala de Bienestar Psicológico de Ryff (tabla 2).

\section{Tabla 2. Bienestar psicológico de los adultos mayores}

Fuente: CENVE, Autores

\begin{tabular}{llllll}
\hline & & & & & Porcentaje \\
& & Frecuencia & Porcentaje & Porcentaje válido & acumulado \\
\hline Válido & Nivel Bajo & 13 & 65,0 & 65,0 & 65,0 \\
\cline { 2 - 6 } & Nivel Moderado & 4 & 20,0 & 20,0 & 85,0 \\
\cline { 2 - 6 } & Nivel Alto & 2 & 10,0 & 10,0 & 95,0 \\
\cline { 2 - 6 } & Nivel Elevado & 1 & 5,0 & Fuente: Escalà de Bienestar Psicológico de Ryff, Autores \\
\cline { 2 - 5 } & Total & 20 & 100,0 & 100,0 & \\
\hline
\end{tabular}

En cuanto al segundo objetivo, se tomó en consideración los datos arrojados por dimensiones Tabla 1. Nivel de Estereotipos negativos hacia la vejez por parte de la Familia

\begin{tabular}{llllll}
\hline & & & & Porcentaje \\
\hline Válido & Nivecuencia & Porcentaje & Porcentaje válido & acumulado \\
\cline { 2 - 6 } & Nivel Moderado & 6 & 15,0 & 15,0 & 15,0 \\
\cline { 2 - 5 } & Nivel Alto & 11 & 30,0 & 30,0 & 45,0 \\
\hline Total & 20 & 55,0 & 55,0 & 100,0 \\
\hline
\end{tabular}

preestablecidas por el CENVE, las cuales obtuvieron valores de frecuencia en Salud (hi=8), MotivacionalSocial (hi=5) y Carácter-Personalidad ( $\mathrm{hi}=7)$, datos que corroborados con el porcentaje presentado en el Gráfico 1, indican la predominancia porcentual del factor Salud. sobre los otros dos factores. 


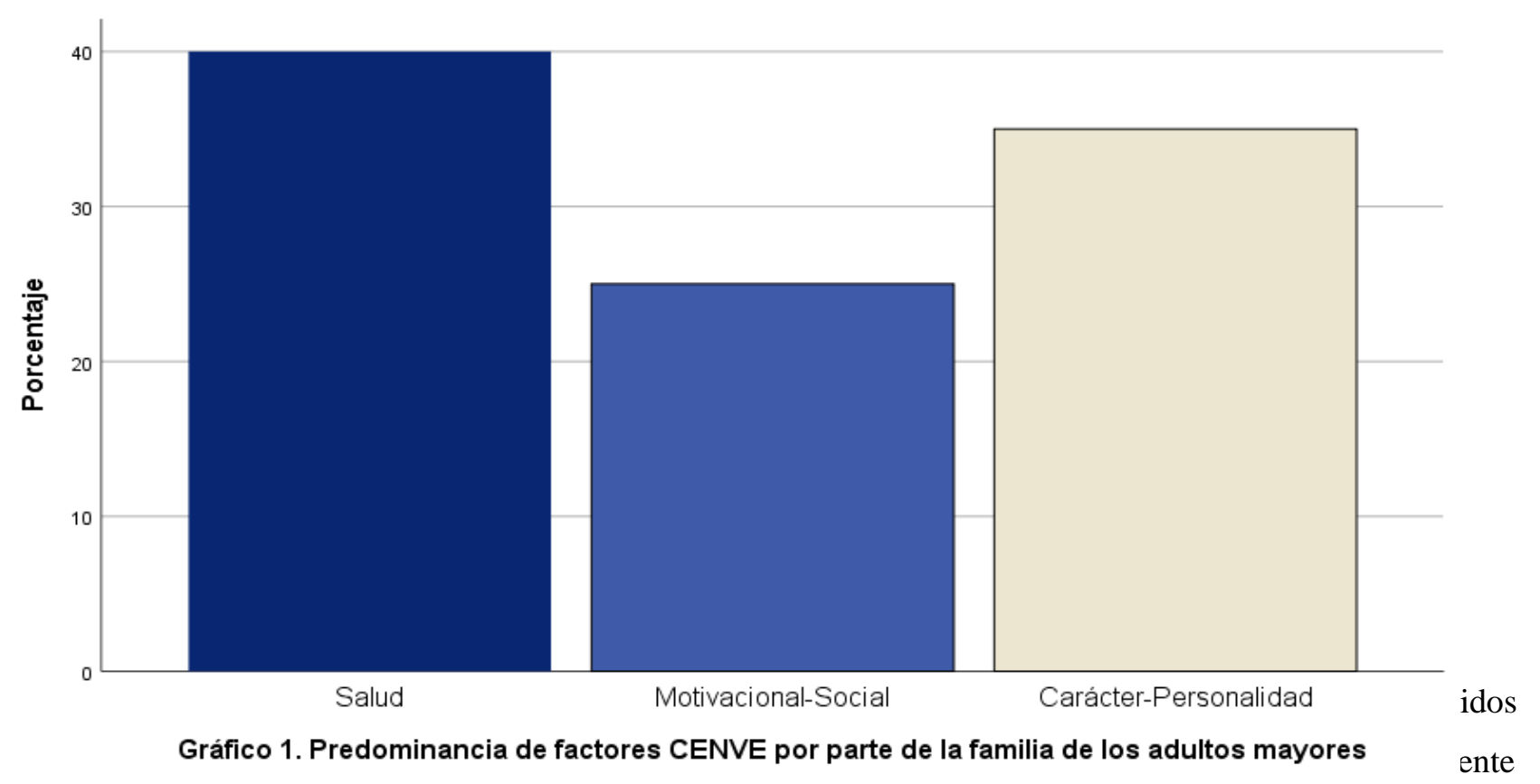

proporcional entre los estereotipos negativos hacia la vejez por parte de la familia y el bienestar psicológico del adulto mayor corroborado con la prueba del Chi cuadrado $\mathrm{x}^{2}(6)=24,248, \mathrm{p}<0,05$, con coeficiente de contingencia $=0,740$ junto con los valores contingentes resumidos en las tablas 4 y 5 . Esta relación señala que las personas que se encuentran con un nivel alto y elevado de bienestar psicológico ( $20 \%$ de la muestra) representan al total de personas que tienen un nivel bajo de estereotipos negativos en la familia, como se evidencia en el gráfico 2.

Tabla 3. Pruebas de chi-cuadrado entre las variables

\begin{tabular}{llll}
\hline & Valor & Df & $\begin{array}{l}\text { Significación asintótica } \\
\text { (bilateral) }\end{array}$ \\
\hline Chi-cuadrado de Pearson & $24,248^{\mathrm{a}}$ & 6 &, 000 \\
\hline Razón de verosimilitud & 20,439 & 6 &, 002 \\
\hline Asociación lineal por lineal & 12,728 & 1 &, 000 \\
\hline N de casos válidos & 20 & - & - \\
\hline a. 11 casillas $(91,7 \%)$ han esperado un recuento menor que 5. El recuento mínimo esperado es , 15. \\
Fuente: CENVE y Escala de Bienestar Psicológico de Ryff, Autores \\
Tabla 4. Medidas simétricas de correlación entre las variables
\end{tabular}


Revista Sinapsis. Vol. 2, Nro 20, diciembre de 2021, ISSN 1390 - 9770

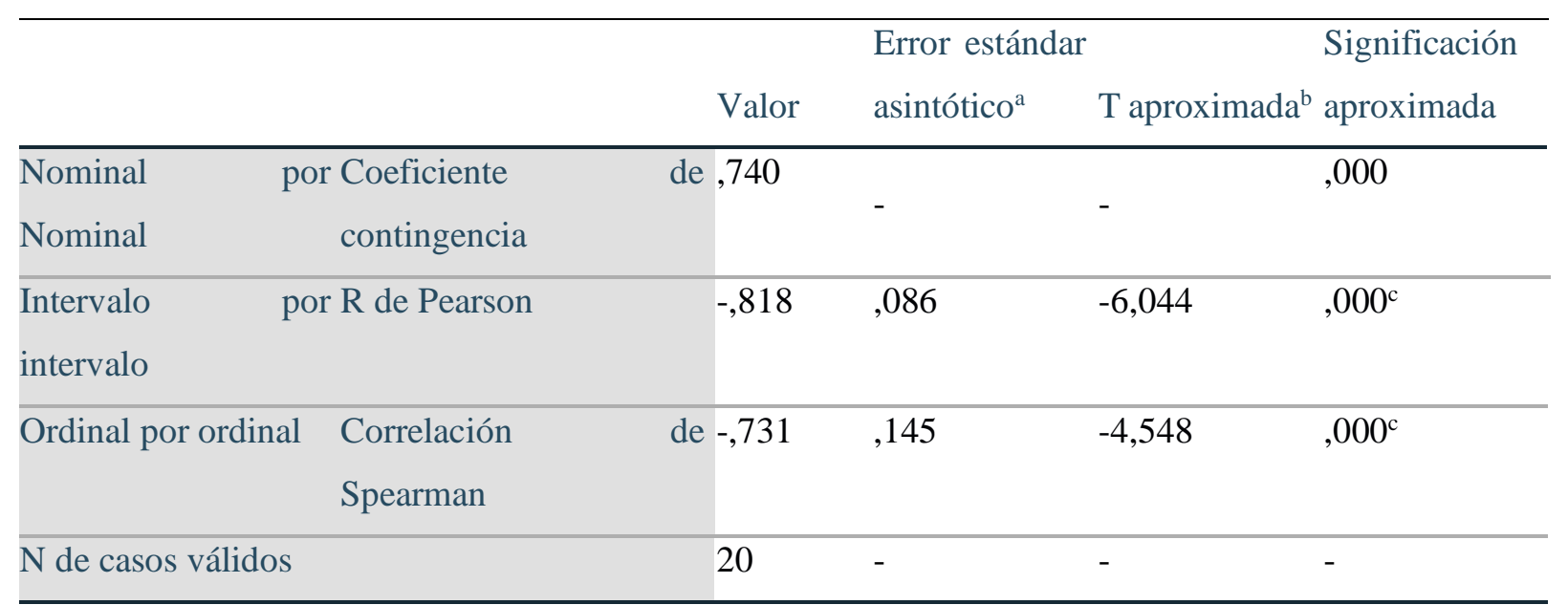

a. No se presupone la hipótesis nula.

b. Utilización del error estándar asintótico que presupone la hipótesis nula.

c. Se basa en aproximación normal.

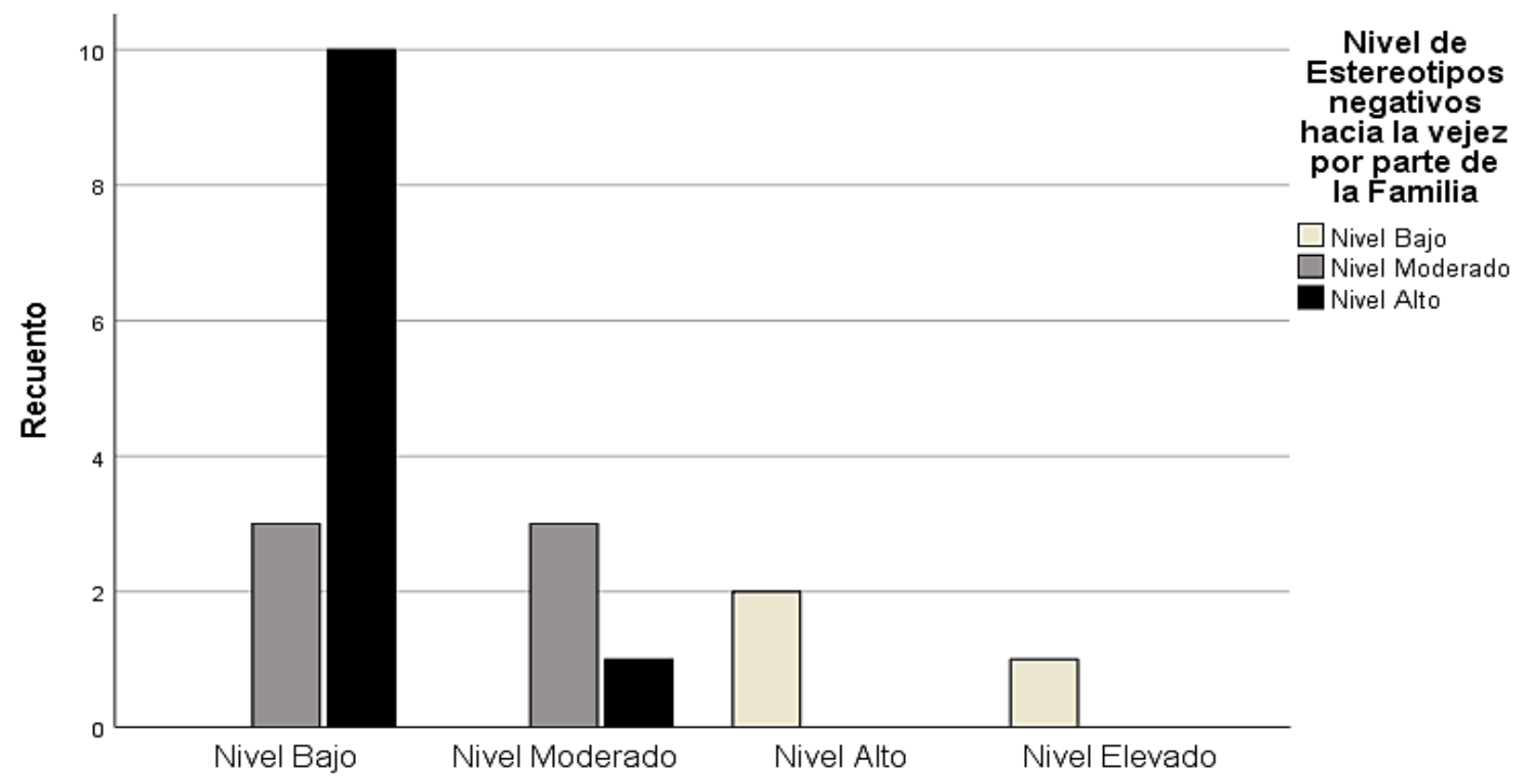

Figura 2. Correlación entre los resultados del CENVE y la Escala de Bienestar Psicológico.

Fuente: CENVE y Escala de Bienestar Psicológico de Ryff, Autores

\section{Discusión}

La investigación se planteó identificar la incidencia de los estereotipos negativos sobre la vejez en la familia sobre el bienestar psicológico de las personas adultas mayores, para ello se analizó las relaciones estadísticas 
entre la puntuación general y factorial obtenidas del Cuestionario de estereotipos negativos hacia la vejez (CENVE) con los resultantes de la Escala de Bienestar Psicológico de Ryff.

En primera instancia se encontró que el 55,0\% de la muestra posee un nivel alto de estereotipos negativos hacia la vejez, porcentaje contradictorio al de Prego \& Solari (2020) realizado en Uruguay en personas cuidadoras formales, en donde la suma porcentual del nivel alto y muy alto de estereotipos negativos fue de 27,3\%, empero en otra investigación realizada por Franco S et al., (2010) con el personal de salud de un hospital en la ciudad de Querétaro en México, se tuvo un porcentaje de 65,0\% de prevalencia de estereotipos negativos.

Estos datos contrastantes podrían corresponder a los diferentes contextos en las que se desarrollaron las investigaciones, el estudio realizado en Uruguay comprendió población con nivel académico alto y con un mínimo de un año en trabajo formal de cuidado al adulto mayor; y en la pesquisa en México fue en estudiantes de enfermería que tenían adultos mayores en su familia conviviente, lo que lleva a inferir que en el presente estudio, el bajo nivel de formación académica en las personas cuidadoras informales (familiares) podría ser un justificante de estas discrepancias, sin sugerir que sea la única causa probable. El análisis de los resultados obtenidos ha arrojado datos a favor de que la puntuación emanada en el CENVE aplicado a la familia conviviente, está estrechamente relacionada con el Bienestar Psicológico percibido por el adulto mayor, reflejándose estadísticamente con la prueba del Chi cuadrado, este resultado concuerda con el de Castillo, (2017) quién en un estudio correlacional-causal entre estereotipos negativos hacia la vejez de cuidadores formales con aspectos psicológicos de los adultos mayores, concluyó que las variables antes mencionadas tienen relación causal.

La comparación factorial sobre los estereotipos negativos hacia la vejez, indica que existió mayor incidencia en el factor Salud, esta visión negativa de la salud de las personas mayores es consistente con la creencia equivocada de que el desgaste del organismo, propio del envejecimiento, conduce inexorablemente a procesos patológicos de enfermedades inhabilitantes y a la dependencia, esto pone en evidencia la hegemonía del enfoque biologicista, que a nivel psicosocial se traduce en una vulnerabilidad circunscrita y forzada, poniendo al adulto mayor en un rol pasivo ante reestructuración del poder en la familia y el papel de beneficiarios de los sistemas de protección social y atención sanitaria. Vale esclarecer según señala (Pinillos, 1994):"“ la vida humana no es solo biológica, sino que además es biográfica e histórica” (p.16). Seguido por el factor Carácter-Personalidad, que acapara las creencias estereotipadas en torno a la involución de las características personológicas, rigidez mental y labilidad emocional (Sánchez, 2004). El factor Motivación-Social se presentó en menor proporción, el cual encasilla creencias adjudicando que los adultos mayores presentan carencias afectivas, falta de intereses vitales y capacidad disminuida para desempeñar actividades laborales o productivas, lo que demarca un debilitamiento del estatus adulto, tal 
como lo plantea Lasagni et al., (2012). La prevalencia factorial del presente estudio se manifiesta de forma similar con los de Franco S et al., (2010b), al menos con el factor Salud como el más incidente, así mismo Duran-Badillo et al., (2016), considerando las condiciones de salud, principalmente la discapacidad física y visual presente en los adultos mayores de la muestra, se podría conjeturar que esta circunstancia está estrechamente relacionada con el reforzamiento y mantenimiento de estereotipos negativos en los familiares cuidadores y no necesariamente a la edad.

Hay que destacar que la investigación presente, a diferencia de otras referidas en contrastación, posee una muestra de adultos mayores con discapacidad establecida, entre las que constan la física y visual, las cuales pudieron ser adquiridas en edad temprana o en adultez, limitando la autonomía en el adulto mayor y condicionando el bienestar psicológico autopercibido, generando una dependencia aún mayor que en personas sin discapacidad, esto se relaciona a lo que manifiesta Dulcey-Ru \& Aldivieso (2002) de que la experiencia de cuidar adultos mayores dependientes forja la generalización entorno a los prejuicios de la dimensión salud, simultáneamente es pertinente referir el llamado Paradigma de Apoyos, el cual da vital importancia al ajuste entre las capacidades de la persona con discapacidad y las demandas del entorno, argumento que respalda la idea principal de que un contexto familiar lleno de estereotipos negativos sobre la vejez, juega un rol sustancial en la percepción del bienestar psicológico en el adulto mayor, debido a los comportamientos y actitudes discriminatorias aparentemente "inofensivas" que derivan de estos esquemas.

\section{Conclusiones}

En línea con los resultados de este trabajo se logra concluir que el nivel de presencia de estereotipos negativos hacia la vejez es particularmente alto, con prevalencia del factor Salud dentro de las dimensiones establecidas por el CENVE, así mismo existe relación proporcional de incidencia de los estereotipos negativos hacia la vejez por parte de la familia con el bienestar psicológico percibido por parte los adultos mayores en la escala de Ryff. Por tales motivos se hace necesario visibilizar los vestigios del contexto familiar en la interacción sea positiva o negativa con el adulto mayor, sin infravalorar la interindividualidad del envejecer. El estudio de la presente temática resulta fundamental para la creación de medidas preventivas e interventivas, con resultados que puedan transmitirse generacionalmente, se sugiere seguir expandiendo las diversas visiones y variables que interceden en el bienestar psicológico del adulto mayor.

\section{Referencias bibliográficas}

Aparicio, M., Sánchez, M., Cuellar, I., Castellanos, B., Fernández, M., \& De Tena, A. (2008). La salud física y psicológica de las personas cuidadoras. https://www.yumpu.com/es/document/read/14563494/la-saludfisica-y-psicologica-de-las-personas-cuidadoras-imserso 
Castillo, J. J. (2017). Aspectos psicológicos relacionados con los estereotipos negativos hacia la vejez en adultos mayores. 29.

Córdoba, A. M. C., Agudelo, J. L. A., \& Ruiz, A. C. S. (2010). Identificación de estereotipos y prejuicios hacia la vejez presentes en una comunidad educativa de florida blanca. Psychologia. Avances de la disciplina, 4(1), 73-87.

Dulcey-Ru, E., \& Aldivieso, C. U. V. (2002). Psicología del ciclo vital: Hacia una visión comprehensiva de la vida humana. 12.

Duran-Badillo, T., Miranda-Posadas, C., Cruz-Barrera, L. G., \& Aguilar-Hernández, R. M. (2016). Estereotipos negativos sobre la vejez en estudiantes universitarios de enfermería. 5.

Estrada, L. C., Riera, R. H., Jiménez, I. P., \& Oca, O. S. M. de. (2008). Caracterización de la atención familiar al adulto mayor. Archivo Médico de Camagüey, 12(6), 1-10.

Franco S, M., Villarreal R, E., Vargas D, E. R., Martínez G, L., \& Galicia R, L. (2010a). Estereotipos negativos de la vejez en personal de salud de un Hospital de la Ciudad de Querétaro, México. Revista médica de Chile, 138(8). https://doi.org/10.4067/S0034-98872010000800007

Franco S, M., Villarreal R, E., Vargas D, E. R., Martínez G, L., \& Galicia R, L. (2010b). Estereotipos negativos de la vejez en personal de salud de un Hospital de la Ciudad de Querétaro, México. Revista médica de Chile, 138(8). https://doi.org/10.4067/S0034-98872010000800007

García, J., \& Ibarra, C. (2018). Bienestar psicológico en adultos mayores institucionalizados y no institucionalizados del Municipio Rionegro. https://repository.ucc.edu.co/bitstream/20.500.12494/16337/1/2019_bienestar_adulto_mayor.pdf

Guerra, A. M. (2014). Bienestar psicológico en los enfermeros y médicos que tratan pacientes con cáncer en el Instituto de Cancerología (INCAN). http://biblio3.url.edu.gt/Tesario/2014/05/42/Guerra-Alesa.pdf

Instituto Nacional contra la Discriminación, la Xenofobia y el Racismo. (s. f.). Discriminación por edad, vejez, estereotipos y prejuicios. 60 .

Lasagni, V., Rodriguez, M., \& Bernal, R. (2012). Estereotipos hacia la Vejez en Adultos Mayores y en Estudiantes Universitarios en diez países de América Latina. Red Latinoamericana de Gerontología. https://www.gerontologia.org/portal/archivosUpload/INVESTIGACION_ESTEREOTIPOS_HACIA_LA_V 
EJEZ_EN_AMERICA_LATINA_2012.pdf?fbclid=IwAR2AKzMpz6Ug8aqkbX3WXe9ps1MQTlfNa7Cd9Z1 xi4beIX-Nd16FAS4O4LI

Montes, de O. V., \& Hebrero, M. (2008). Dinámica familiar, envejecimiento y deterioro funcional en México_. São Paulo, 24.

Ortiz Arriagada, J. B., \& Castro Salas, M. (2009). Bienestar psicológico de los adultos mayores, su relación con la autoestima y la autoeficacia: Contribución de enfermería. Ciencia y enfermería, 15(1). https://doi.org/10.4067/S0717-95532009000100004

Pabón, D. K. P., \& Delgado, J. A. D. (2017). Maltrato en la población adulta mayor: Una revisión. Espacio Abierto, 26(2), 245-267.

Pinillos, J. L. (1994). Mitos y estereotipos en los mayores que vienen. Una aproximación pluridisciplinar al entorno de la vejez. Fundación Caja de Madrid.

Rodríguez, P. A. V. M. V. (2019, abril 3). Sentimientos y prejuicios del adulto mayor respecto al envejecimiento. CienciAcierta. http://www.cienciacierta.uadec.mx/2019/04/03/sentimientos-y-prejuicios-deladulto-mayor-respecto-al-envejecimiento/

Sánchez, C. (2004). Estereotipos negativos hacia la vejez y su relación con variables sociodemográficas, $\begin{array}{lllll}\text { psicosociales } & y & \text { psicológicas } & \text { [Universidad } & \text { Málaga]. }\end{array}$ http://www.biblioteca.uma.es/bbldoc/tesisuma/16704046.pdf

Valhondo, A. M., Rubio, C. F., \& Castro, P. G. (s. f.). Familia y envejecimiento, disfunciones y programas psicoterapéuticos. 15.

Velasco, V., Suárez, G. G., Limones, M. de L., Reyes, H., \& Delgado, V. E. (2018). Estereotipos del adulto mayor hacia el envejecimiento. Journal of Geriatrics, 2018, 1-9. https://doi.org/10.1155/2015/954027 